\title{
Airborne Visible / Infrared Imaging Spectrometer AVIS: Design, Characterization and Calibration
}

\author{
Natascha Oppelt * and Wolfram Mauser \\ Ludwig-Maximilians-Universität München, Dept. for Geography, Luisenstr. 37, 80333 Munich, \\ Germany. \\ E-mail: n.oppelt@1mu.de; w.mauser@iggf.geo.uni-muenchen.de \\ * Author to whom correspondence should be addressed.
}

Received: 31 July 2007 / Accepted: 13 September 2007 / Published: 14 September 2007

\begin{abstract}
The Airborne Visible / Infrared imaging Spectrometer AVIS is a hyperspectral imager designed for environmental monitoring purposes. The sensor, which was constructed entirely from commercially available components, has been successfully deployed during several experiments between 1999 and 2007. We describe the instrument design and present the results of laboratory characterization and calibration of the system's second generation, AVIS-2, which is currently being operated. The processing of the data is described and examples of remote sensing reflectance data are presented.
\end{abstract}

Keywords: imaging spectroscopy, environmental monitoring, airborne hyperspectral sensor

\section{Introduction}

There is growing interest in hyperspectral remote sensing for research and applications in a variety of fields, including geology, agriculture, forestry, coastal and inland water studies, environmental hazards assessment, and urban studies [1,2]. The concept of hyperspectral imaging originated from geological applications in the early 1980s, mainly for the purpose of mineral exploration [3]. Imaging spectrometers acquire data in many contiguous narrow bands leading to a complete reflectance or emittance spectrum for each pixel of the wavelength region covered.

Until recently imaging spectroscopy was an expensive tool due to the limitations to airborne systems. Existing airborne hyperspectral sensors such as AVIRIS (Airborne Visible / InfraRed 
Imaging Spectrometer), casi (compact airborne spectral imager) or HYMAP (HYperspectral MAPping) are one-of-a-kind sensors supported by large, dedicated programs. They have proven useful for many applications, but are difficult to schedule due to many competing user requirements. Therefore airborne imaging spectrometer systems are cost-intensive for the user and the availability for multi-temporal applications is low. A high temporal resolution is required for environmental analysis because most developments either in natural or in managed ecosystems can only be monitored through time series of remote sensing observations. In 1997 these drawbacks led to the idea of a compact lightweight imaging spectrometer to overcome the difficulties of the existing systems and provide data for the environmental research purposes of the Department for Geography of the Ludwig-MaximiliansUniversity in Munich (Germany). This was the birth of the AVIS (Airborne Visible / Infrared imaging Spectrometer) system.

The ongoing development of digital imaging tools enabled the enhancement of the first generation of AVIS to AVIS-2. Dedicated software was developed to acquire and process the data. Since 2001 AVIS-2 is being operated and will form the basis of this paper.

\section{System Specifications}

The specifications of the AVIS system were determined by the availability and affordability of components resulting in a Charged Coupled Device (CCD)-based system operating in the visible (VIS) to near infrared (NIR) spectral domain. AVIS is conceived to be operated on different platforms such as Dornier DO-27, DO-228 and microlight aircrafts, where the sensor is mounted on vibration dampening mounts.

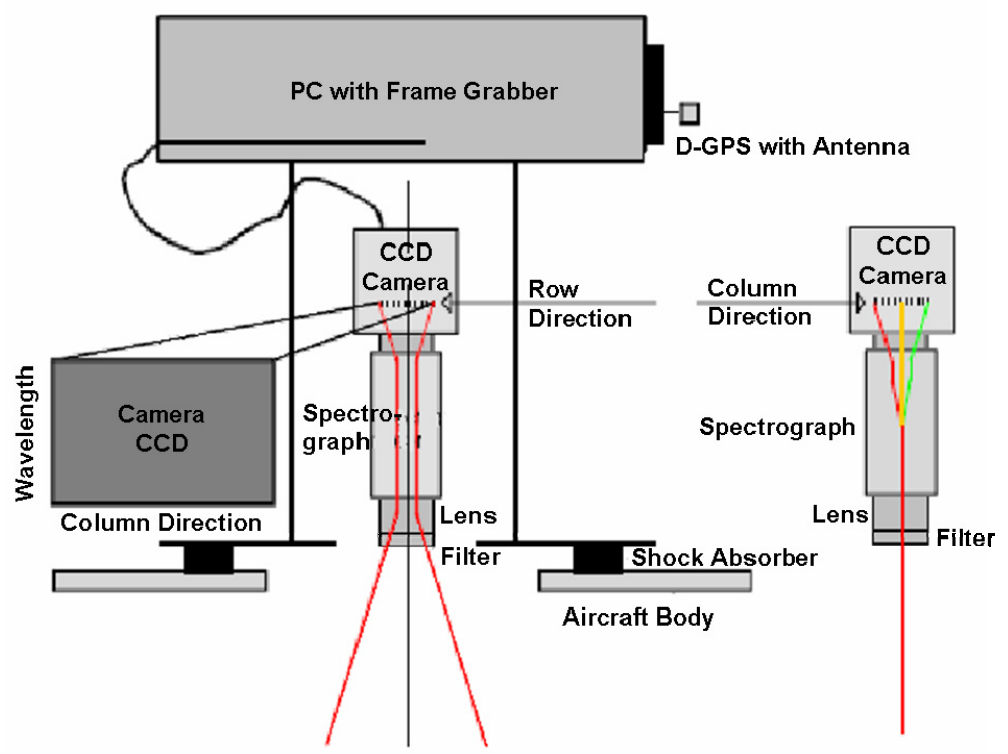

Figure 1. Components of AVIS-2 [4, modified].

The components of the system are shown in Figure 1. AVIS-2 is based on a spectrograph (IMSPECTOR, Specim Inc.), which is mounted onto a black and white 2/3" CCD-video camera (VOSSKÜHLER 1300QLN) and a filter-lens system (f/1.4, $8 \mathrm{~mm}$; SCHNEIDER Optics). Radiation which enters the spectrograph slit is collimated by the first lens and refracted at the prism surface to 
the correct incident angle of the holographic grating (Figure 2). The grating disperses the light according to the common grating Equation 1 [5].

$$
s \cdot \sin \theta_{m}=m \cdot \lambda
$$

where

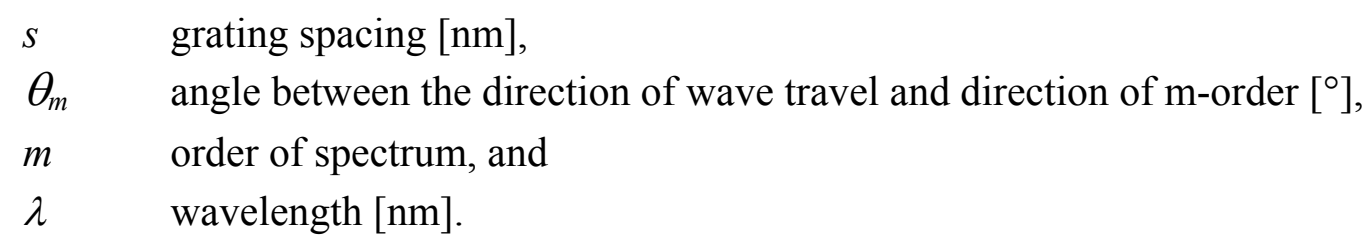

Any tubular optics are sensitive to ghost images and scattered light caused by reflections at optical surfaces. There is also a risk of light scattering caused by transmitted, unwanted orders of diffraction in the holographic grating. These are taken into account by using baffles inside the tube as well as multilayer coatings of all air/glass surfaces. The tilted surfaces of the prisms refract remaining forwarded reflections to blackened surfaces of the spectrometer tube leading to a distortion due to scattered light of less than $1 \%[5,6]$.

Spatial information at the entrance slit is transferred to the imaging plane along the axis parallel to the slit length direction (spatial axis in Figure 2). The output of the spectrograph is a column of 512 pixels (spectral axis in Figure 2) representing the wavelengths from 400 to $900 \mathrm{~nm}$ with a spectral sampling rate of $3.7 \mathrm{~nm}$. The spatial output of the spectrograph is a row of 640 pixels.
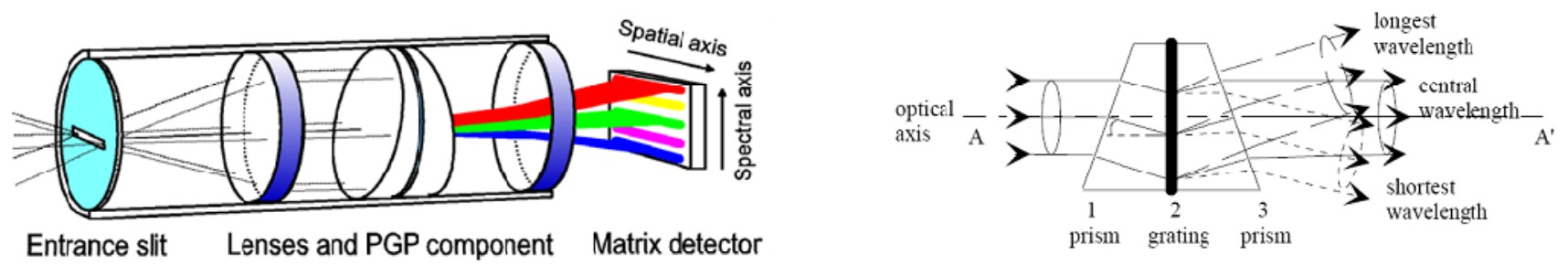

Figure 2. Schematic diagram of spectrograph ImSpector (left) and basic principle of the direct-vision element (right) [5].

The spectrograph is mounted onto a 2/3" CCD-camera (VOSSKÜHLER 1300QLN). The primary selection criteria for the camera were a progressive interline-transfer sensor and asynchronous operation mode (image on demand) which enables very short exposure times up to $1 / 10000 \mathrm{~s}$ with full resolution (1280 x 1024 pixel). This enables the monitoring of moving targets or the monitoring of fixed targets with the camera being moved. When the system is moved above ground, a twodimensional flight line is created. The high resolution output of the camera is binned to a $640 \times 512$ image to increase the signal-to-noise ratio. Data rates are typically less than $15 \mathrm{MB} / \mathrm{s}$, but several gigabytes may be stored during a flight line. The data is viewed in real-time on the monitor to check data quality. 
A Microsoft Windows NT-based personal computer (PC) controls the camera through a PCI interface card. Software was developed to configure the camera for the particular mode desired, including gain, frame rate, and number of frames to collect. The signal is digitized via a frame grabber and stored in a standard file format on a hard disk of the connected PC together with additional data from a differential Global Positioning System (dGPS, GARMIN) and an Inertia Navigation System (INS, iMAR GmbH), which are mounted on the same plate as the camera. The dGPS/INS data are stored in the header of each image line and allow a line-by-line positioning during the geometric correction of the images (section 3.3.3).

\section{Calibration and Processing}

Pushbroom type imaging spectrometers offer advantages in robustness, integration time, speed, and spectral/spatial resolution. Nevertheless, they also suffer from some intrinsic sources of malfunction or defects, which have to be addressed by appropriate correction schemes. Malfunctions inherent to the system or temporally consistent sensor defects can be analyzed in the laboratory. Correction schemes can therefore be implemented in the radiometric or spectral processing of the data. This has to be carried out each time the system composition or system settings are changed. The calibration and processing chains necessary for an appropriate evaluation of the AVIS-2 data are presented in Figure 3 and will be described in more detail in the following sections.

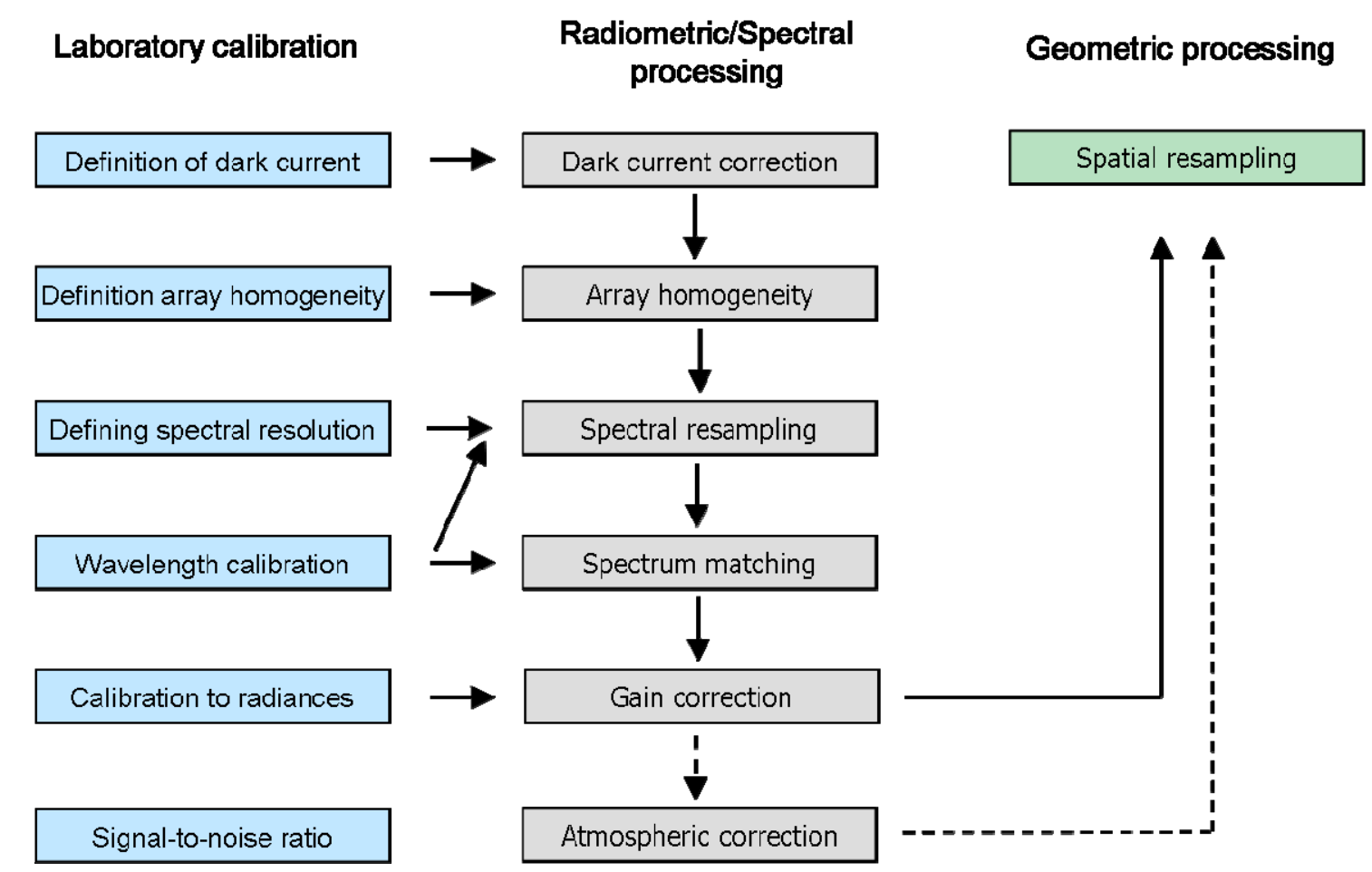

Figure 3. Processing chains for laboratory calibration and data processing chains. 


\subsection{Radiometric properties and calibration}

The analysis of a sensor's radiometry provides information essential for the handling of imaging spectrometer data. The radiometric resolution is one of the most dominant features of radiometric properties because it defines the number of grey values into which the incoming radiance can be resolved. The radiance measured by a system is influenced by factors such as changes in scene illumination, atmospheric conditions, viewing geometry, and instrument characteristics. The temporally consistent characteristics of AVIS-2 are analyzed and corrected during the processing of the data. These characteristics are described in this paper including the analysis of dark current, homogeneity of the CCD, and the signal-to-noise ratio.

\subsubsection{Radiometric resolution}

The radiometric resolution of a system is limited by the lowest radiometric resolution of its components. The camera offers 12 bit imaging on an array of $1280 \times 1024$ pixels and a 14 bit imaging on $640 \times 512$ pixels via two by two binning. By using the $640 \times 512$ pixel mode and additional software binning by a factor of four in the spectral direction, an effective radiometric resolution of 15 bits can be achieved. The nominal spatial resolutions of the acquired images are reduced to 640 pixels in the spatial direction and 128 pixels in the spectral dimension. Besides the increase of bit depth the advantage of binning is the reduction of noise. Furthermore binning enables the camera to increase the image rate from 12.5 frames/s to 25 frames/s.

\subsubsection{Dark current}

Dark current is caused by thermally generated electrons within the camera. The dark current density can be analyzed by measuring the spectral response for a given temperature with closed aperture. For AVIS-2 the dark current is a constant offset (binned DN =1180), which is subtracted pixel by pixel from the original data during pre-processing (Equation 2).

$$
D N_{d c c}=D N_{o r g}-D N_{d a r k}
$$

where

$D N_{d c c} \quad$ dark current corrected grey value [digital number],

$D N_{\text {org }} \quad$ original grey value [digital number], and

$D N_{\text {dark }} \quad$ dark current [digital number].

\subsubsection{Spatial uniformity}

Spatial uniformity is the radiometric response equality within a frame. Array detectors such as CCD elements contain defects. These defects are regions of reduced sensitivity or increased dark current. For scientific applications these variations affect results and therefore must be corrected [6]. 
Another aspect is vignetting, which occurs when the frame of the lens shades the radiation with high incident angles.

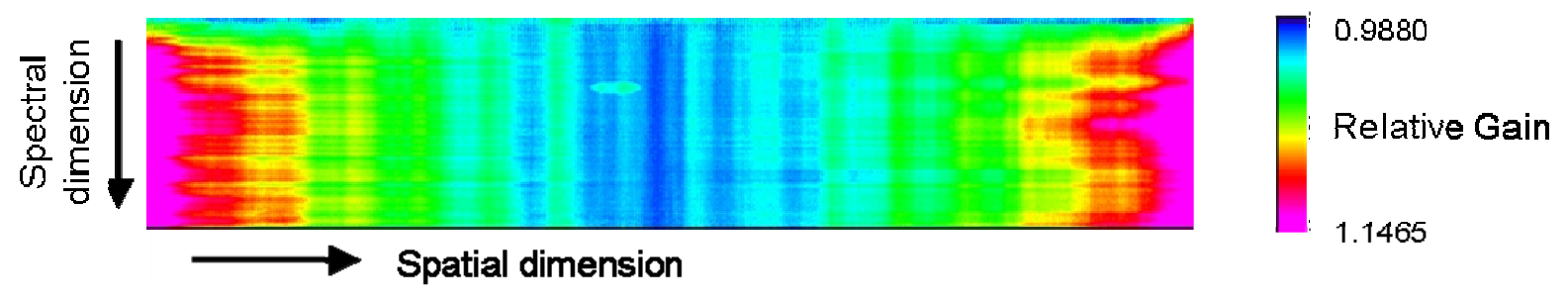

Figure 4. AVIS-2 relative gain matrix.

The relative sensitivity of the CCD is measured under homogeneous light conditions using an integrating sphere as an inherently stable illumination source. A Tungsten Halogen lamp is used as the illumination source. Figure 4 presents the relative gain matrix that was derived from these measurements. The most prominent features are the vertical striping which is superimposed on a general decrease of sensitivity towards the left and right edges of the CCD caused by the vignetting effect.

These variations form a pattern that is constant over time and can be corrected by multiplying each pixel with its corresponding relative sensitivity: pixels with reduced sensitivity are multiplied with a high gain factor $(>1)$ while high sensitivity regions are multiplied with a low gain factor $(<1)$.

\subsubsection{Signal-to-noise ratio (SNR)}

Once the data is corrected for dark current and array homogeneity, the SNR analysis can be carried out. This is done in the laboratory using the same illumination equipment as described above. The SNR of the AVIS-2 system at full illumination, as defined in Equation 3, can be rated at a maximum of $66 \mathrm{~dB}(2000: 1)$ for the unbinned data.

$$
S N R=\frac{\left(D N_{\text {sat }}-D N_{\text {dark }}\right)}{\sigma_{\text {dark }}}
$$

where
$D N_{\text {sat }} \quad$ grey value just before saturation [digital number], and
$\sigma_{\text {dark }} \quad$ standard deviation of the dark current image.

The SNR in the image data depends on the illumination conditions, the exposure time and the targets monitored. Examples of a vegetated surface (soccer field) and bare soil are presented in Figure 5. 

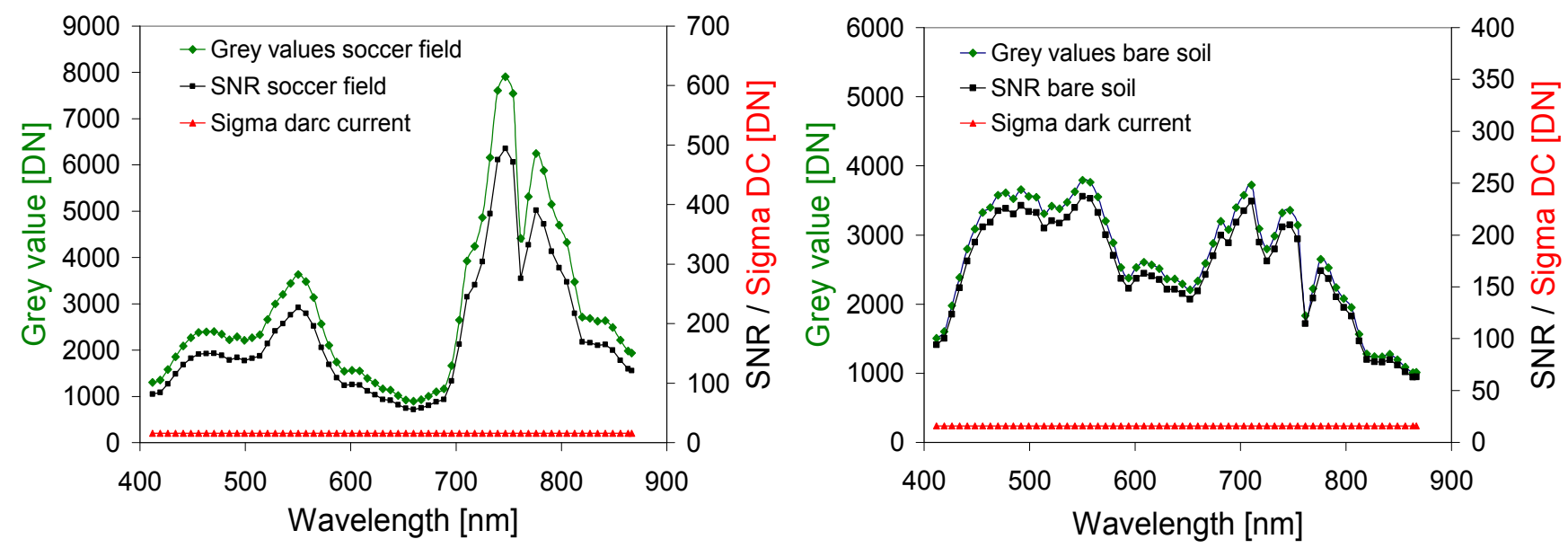

Figure 5. Grey value spectrum and corresponding SNR of AVIS-2 for measurements of a soccer field (left) and bare soil (right) acquired on August 30, 2005.

\subsection{Spectral properties and calibration}

In the discussion of spectral properties of imaging spectrometers the spectral resolution, spectral sampling interval and centre wavelengths are the most important parameters. Assuming a Gaussian shaped response function, the spectral resolution is defined as the full width half maximum (FWHM) of the function. The center wavelength is the peak response of the spectrometer to an infinitesimally narrow emission line [7, 8]. The spectral sampling interval is the distance between two adjacent center wavelengths. Other effects which have to be considered are the analysis of spectral uniformity and responsivity.

\subsubsection{Spectral uniformity}

Due to the intrinsic light dispersion properties of grating spectrometers and to minor misalignment of optical components, the wavelengths for pixels near the center of an array and those near the edges of the same array can be slightly different. This is often referred as the "smile" or "frown" effect [9], which also occurred in the AVIS-2 data. The AVIS-2 frown is illustrated in Figure 6 by means of the varying position of the oxygen absorption resulting in a change of the absorption between two adjacent spectral bands with a maximum shift of $3.96 \mathrm{~nm}$.

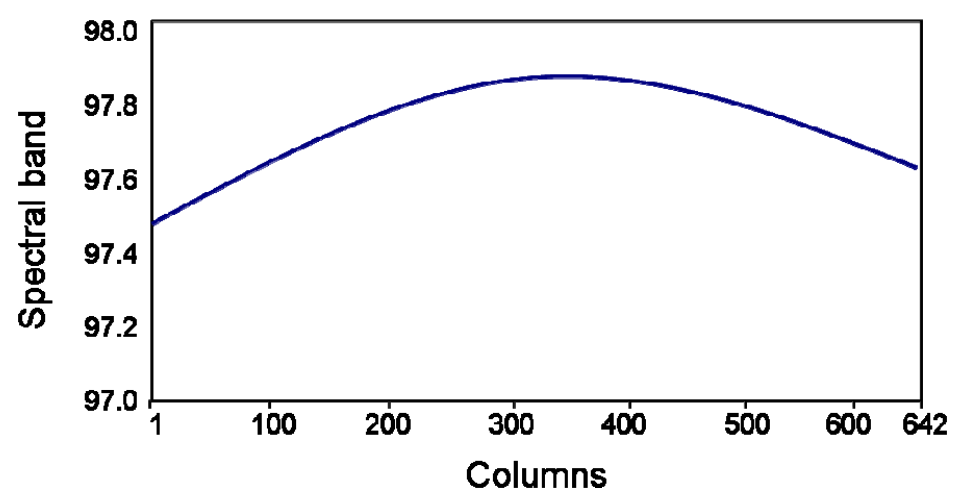

Figure 6. Position of oxygen absorption. 
To correct this effect, an oxygen-fitting algorithm is applied: six spectral bands, which are located around $761 \mathrm{~nm}$, are searched for their absolute minimum, which is then set to a predefined spectral band.

\subsubsection{Spectral Resolution}

The nominal spectral resolution of the spectrograph is $7 \mathrm{~nm}$ [5]. To check this specification the real spectral resolution was measured by correlating measured and modeled at-sensor radiances of the oxygen absorption around $760 \mathrm{~nm}$. The oxygen absorption is a suitable feature because of its characteristic shape with two adjacent absorption peaks (Figure 7).

Therefore, at-sensor radiances were simulated for skylight using MODTRAN 4.2 [6, 7] with different spectral resolutions $(0.5$ to $10 \mathrm{~nm}$ in steps of $0.5 \mathrm{~nm})$. These simulated radiances were compared with AVIS-2 measurements of skylight resulting in a best fit for a spectral resolution of 9 $\mathrm{nm}$. Therefore the real spectral resolution can be defined as $9 \mathrm{~nm}$. Knowing the band number of the oxygen absorption and the spectral resampling rate the actual wavelength range of AVIS-2 can be identified as covering the spectral region from $404 \mathrm{~nm}$ to $875 \mathrm{~nm}$.

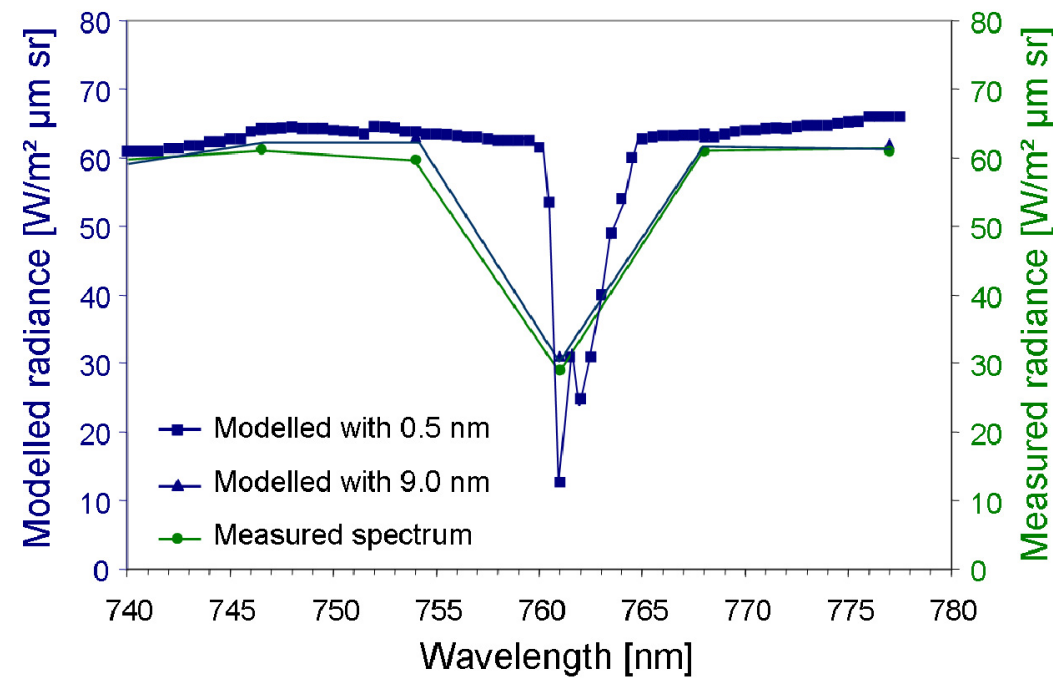

Figure 7. Comparison of simulated MODTRAN radiances and AVIS-2 measurements of skylight.

\subsubsection{Spectral sampling and resampling}

AVIS-2 covers the wavelength region between 404 and $875 \mathrm{~nm}$ with a sampling interval of $3.7 \mathrm{~nm}$. With an actual spectral resolution of $9 \mathrm{~nm}$, the system is oversampled by the factor 2.4.

Therefore the data were resampled to a spectral sampling rate corresponding closely to the spectral resolution. The averaging was done assuming a Gaussian-shaped response function represented by the different weights for the adjacent bands: 


$$
D N_{\text {resampled }}=\frac{A_{1} \cdot D N_{2 i-1}+A_{2} \cdot D N_{2 i}+A_{3} \cdot D N_{2 i+1}}{\sum_{n=1}^{3} A_{i}},
$$

where

$\begin{array}{ll}A_{1} & 0.5, \\ A_{2} & 1.0, \text { and } \\ A_{3} & 0.5 .\end{array}$

By incrementing $i$ a total of 64 resampled spectral bands with a nominal bandwidth of $7.3 \mathrm{~nm}$ is produced from the original 128 AVIS-2 bands. This enables the reduction of the amount of data as well as an increase of the SNR without any loss of spectral information.

\subsubsection{Wavelength calibration}

An accurate wavelength calibration is essential for the retrieval of land or ocean surface reflectances. Especially as the atmospheric gas absorption features are very sharp, errors in wavelength calibration can produce significant errors in the resulting reflectances around these features. A wavelength calibration of the spectrograph had already been carried out by the manufacturer. The center wavelengths changed during resampling, therefore the newly defined wavelengths must be checked. This is done using atomic emission lines from a discharge lamp because most atomic emission lines are known with an accuracy exceeding the requirements of calibration $[9,12]$. The measurements were performed in the darkened laboratory using a krypton lamp as the illumination source. Before analysis the correction for dark current and spatial uniformity was performed as well as the resampling to 64 spectral bands. The results presented in Figure 8 confirm that resampling procedure does not affect the correct wavelength assignment. The deviations turned out to be smaller than the original sampling interval of $3.8 \mathrm{~nm}$.

According to the resampling and wavelength assignment it can be concluded that AVIS-2 operates in the wavelength region between $404 \mathrm{~nm}$ and $875 \mathrm{~nm}$ with a spectral resolution of $9 \mathrm{~nm}$ and a spectral sampling interval of $7.3 \mathrm{~nm}$.

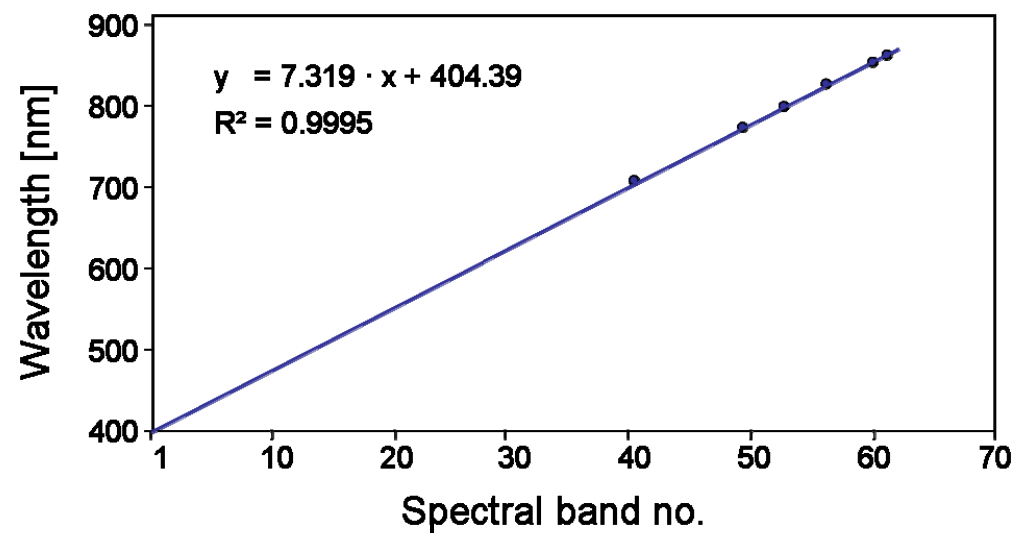

Figure 8. Wavelength calibration using emission lines of a krypton lamp. 


\subsubsection{Spectral responsivity}

The spectral responsivity of AVIS-2 is the result of the responsivities of its components (spectrograph, camera, lens and filter). The spectral responsivity can be analyzed in the laboratory using an integrating sphere and a halogen calibration lamp (OCEAN OPTICS HL-2000) as the illumination source. The intensity of the output from the sphere is determined by performing a transfer calibration from the calibration lamb. The resulting overall efficiency of light collection by the system is highest between 470 and $570 \mathrm{~nm}$ and around $720 \mathrm{~nm}$, which results in a calibration gain that is higher at the wavelengths in the blue, red and towards the NIR (Figure 9).

The data are used to calculate a relationship between grey values and at-sensor radiances for each spectral band according to Equation 5 .

$$
S R_{i}=\left(D N_{i}-D N_{\text {dark }, i}\right) \cdot g_{i}
$$

where

$S R_{i} \quad$ spectral at-sensor radiance for band $i\left[\mathrm{~W} / \mathrm{m}^{2} \mathrm{sr} \mathrm{nm}\right]$,

$D N_{i} \quad$ grey value for band $i[\mathrm{DN}]$,

$D N_{d a r k, i}$ dark current offset for band $i[\mathrm{DN}]$, and

$g_{i} \quad$ spectral sensitivity calibration gain [W/ $\left./ \mathrm{m}^{2} \mathrm{sr} \mathrm{nm}\right]$.

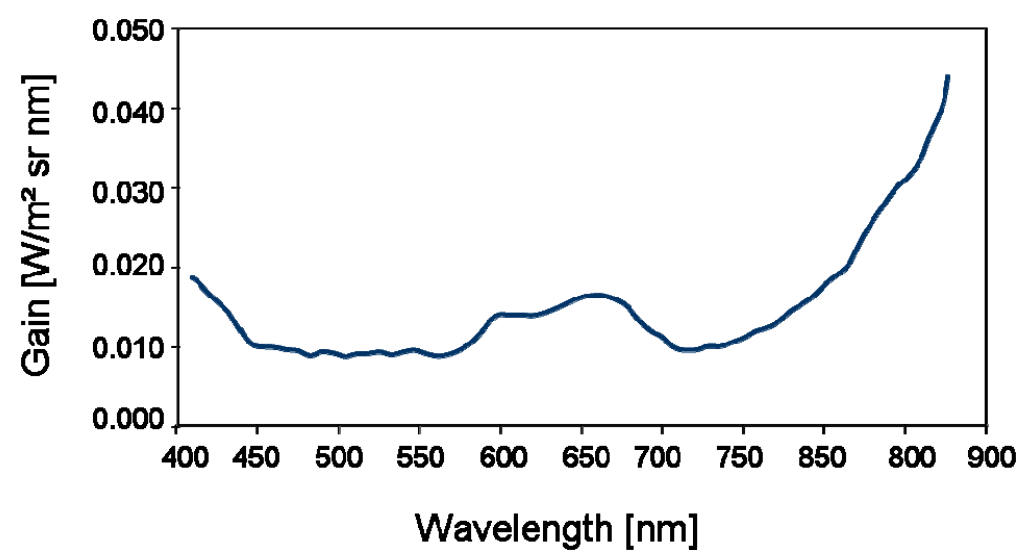

Figure 9. Spectral sensitivity calibration gain.

\subsection{Geometric properties and correction}

The main parameters of interest concerning the geometry are the spatial resolution of the imagery, the field of view (FOV) and the instantaneous field of view (IFOV). The spatial resolution and the FOV depend strongly on the flight altitude, while the IFOV is constant. The IFOV of a system depends on both the spectrograph and the camera.

Remote sensing data corrections in the spatial domain are well known for image registration and rectification processes $[13,14]$. In satellite remote sensing, the processing normally affects the spatial 
dimension of the imagery and is usually to be performed within a distance of 0.1 to 2 pixels. Airborne imagery may have more distinct distortions due to dynamic platform movements caused by turbulence in the lower atmosphere.

A major precondition for successful geometric resampling is that the data is radiometrically corrected and calibrated to physical units (e.g. radiance) [12, 13].

\subsubsection{Spatial resolution and ground sample distance}

In general the spatial resolution on ground $(\mathrm{R})$ depends strongly on the altitude of the sensor. The spatial resolution of airborne imagery can differ between an image line (cross track) and the movement of the aircraft (along track). Therefore both resolutions have to be calculated separately. The former is determined by the camera pixel size and the point spread size of the optics, which is $30 \mu \mathrm{m}$ corresponding to a modular transfer function of 15 line-pairs $/ \mathrm{mm}$. With an array dimension of $8.8 \mathrm{~mm}$, the point spread size limited resolution is $8800 / 30 \cong 300$ points [5]. The resulting spatial resolution on ground can be calculated according to Equation 6 using the altitude of the aircraft $(h)$. The latter is determined by the line width of the system $(\mathrm{W}=2.98 \mathrm{mrad})$ and the altitude (see Equation 7$)$.

$$
\begin{aligned}
& R_{(\text {cross track })}=\frac{h}{300} \\
& R_{(\text {along track })}=W \cdot h
\end{aligned}
$$

According to Equation 8 the ground sample distance (GSD) cross track is always determined by the number of pixels of the camera. The along track sampling depends on the frame rate $(i)$ and the aircraft speed $(v)$ (see Equation 9).

$$
\begin{aligned}
& G S D_{(\text {cross track })}=\frac{h}{640} \\
& G S D_{(\text {along track })}=\frac{v}{i}
\end{aligned}
$$

\subsubsection{Field of view and instantaneous field of view}

The field of view (FOV), normally expressed as the cone angle within which incident radiation is focused on the detector, is determined by the lens. The AVIS-2 system uses an IR compensated (400$1000 \mathrm{~nm}$ ) lens with a FOV of $1.16 \mathrm{rad}$ or $66^{\circ}$.

The instantaneous field of view represents the angle viewed by the CCD for each pixel. Comparable to the spatial resolution of an airborne instrument, it can be distinguished between the IFOV along an image line and the IFOV in the direction of aircraft motion. 
The IFOV along an image line (IFOV $\left.V_{\text {cross track }}\right)$ is determined by the FOV of the lens and the number of pixels per image line $\left(N_{c}=640\right)$ according to Equation 10 . The resulting $I F O V_{\text {cross }}$ track for AVIS-2 is $1.81 \mathrm{mrad}$ before binning.

$$
I F O V_{\text {cross track }}=\frac{F O V_{\text {lens }}}{N_{c}}
$$

The IFOV in direction of the aircraft movement (IFOV $V_{\text {along track }}$ ) is determined by the width of the spectrograph slit $\left(W_{s}=13 \mu \mathrm{m}\right)$ and the focal length of the camera $(f=8.4 \mathrm{~mm})$. According to Equation 11 it can be rated at $1.55 \mathrm{mrad}$.

$$
\text { IFOV } V_{\text {along track }}=\frac{W_{S}}{f}
$$

\subsubsection{Geometric correction}

A dGPS/INS system provides accurate data for geometric processing of the data. The aircraft location (latitude, longitude and altitude) and pointing information (roll, pitch and yaw) are measured with an accuracy of $1.2 \mathrm{mrad}$ at a frequency of $40 \mathrm{~Hz}$.
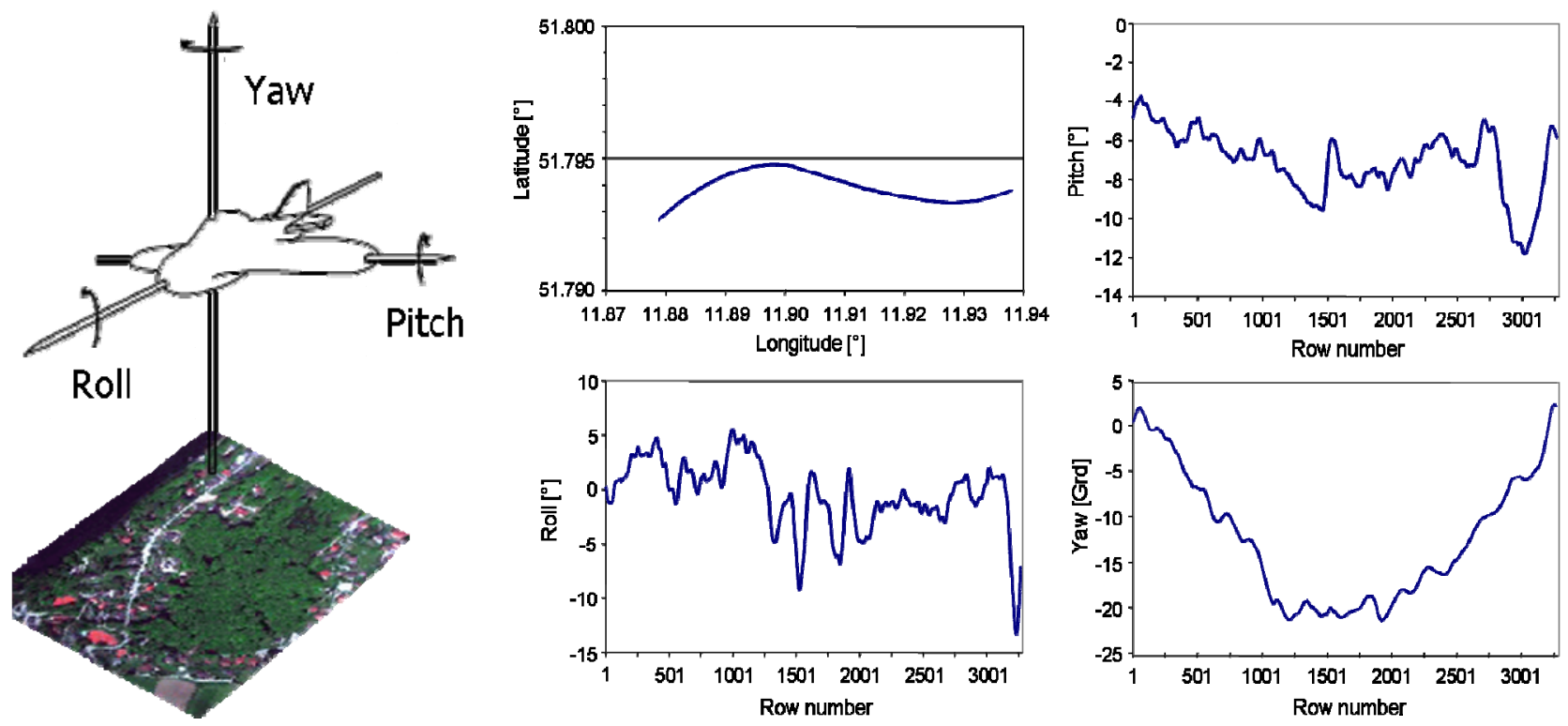

Figure 10. Definition of roll, pitch and yaw (left) and dGPS/INS recordings during a flight line (right).

Figure 11 demonstrates the results of the geometric processing of original flight data using INS/GPS data. The INS/GPS data together with a digital terrain model makes it possible to compensate for the motion of the aircraft and at the same time produces an image that closely matches WGS-84 UTM projection. The image was acquired at a flight altitude of $1450 \mathrm{~m}$ above ground with a ground speed of $25 \mathrm{~m} / \mathrm{s}$ and a frame rate of $12 \mathrm{images} / \mathrm{s}$. 

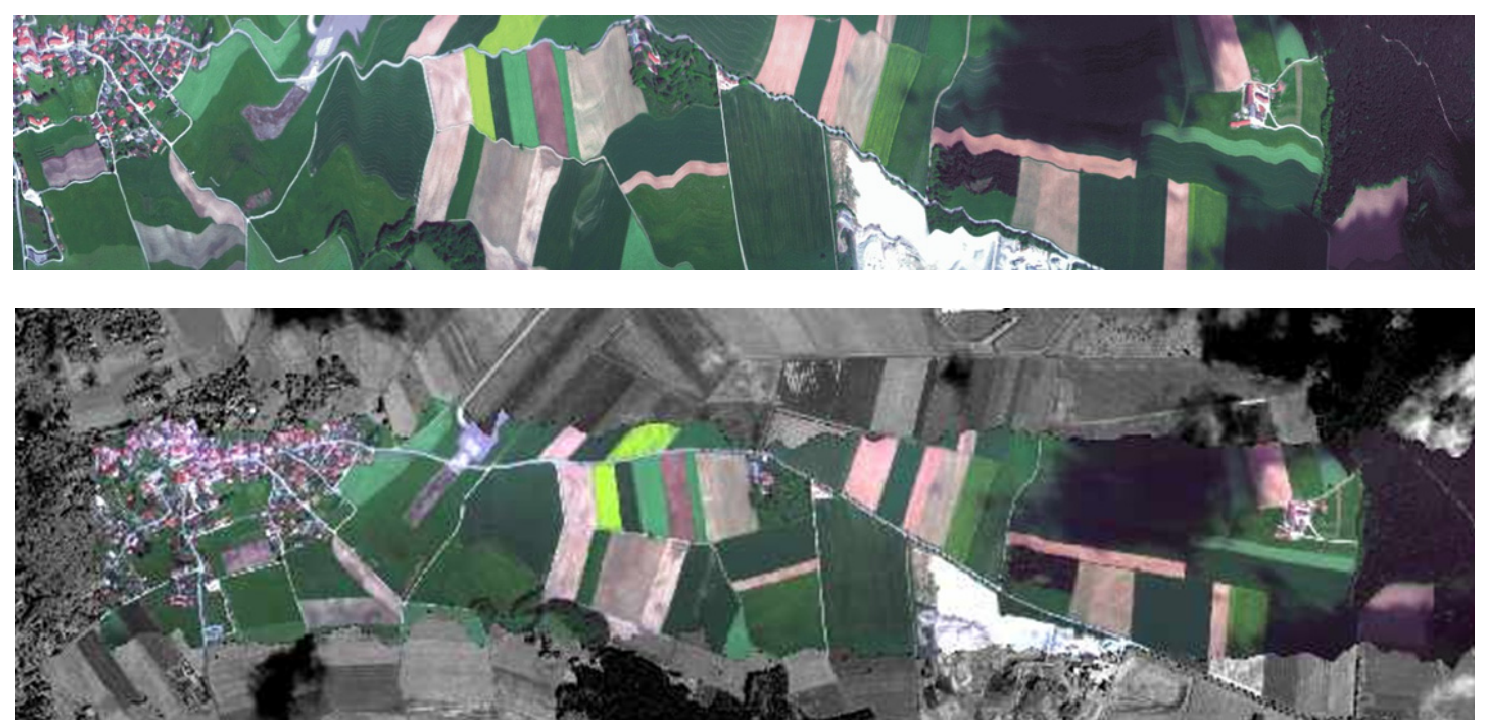

Figure 11. Original (top) and geometrically corrected AVIS-2 data using dGPS/INS attitude information (bottom, overlay with georeferenced IKONOS image).

\section{Examples for the application of AVIS-2 data}

The sensor AVIS-2 was/is used for various applications in agriculture $[4,15,16,17,18]$, floristic studies [19, 20, 21], inland water applications [22] as well as for studies funded by the European Space Agency [23, 24, 25]. Some examples for AVIS-2 applications will be given in the following section.

\subsection{Derivation of reflectance spectra}

Figure 13 shows a flight line acquired with AVIS-2 mounted on a microlight plane, which can be observed in Figure 12.
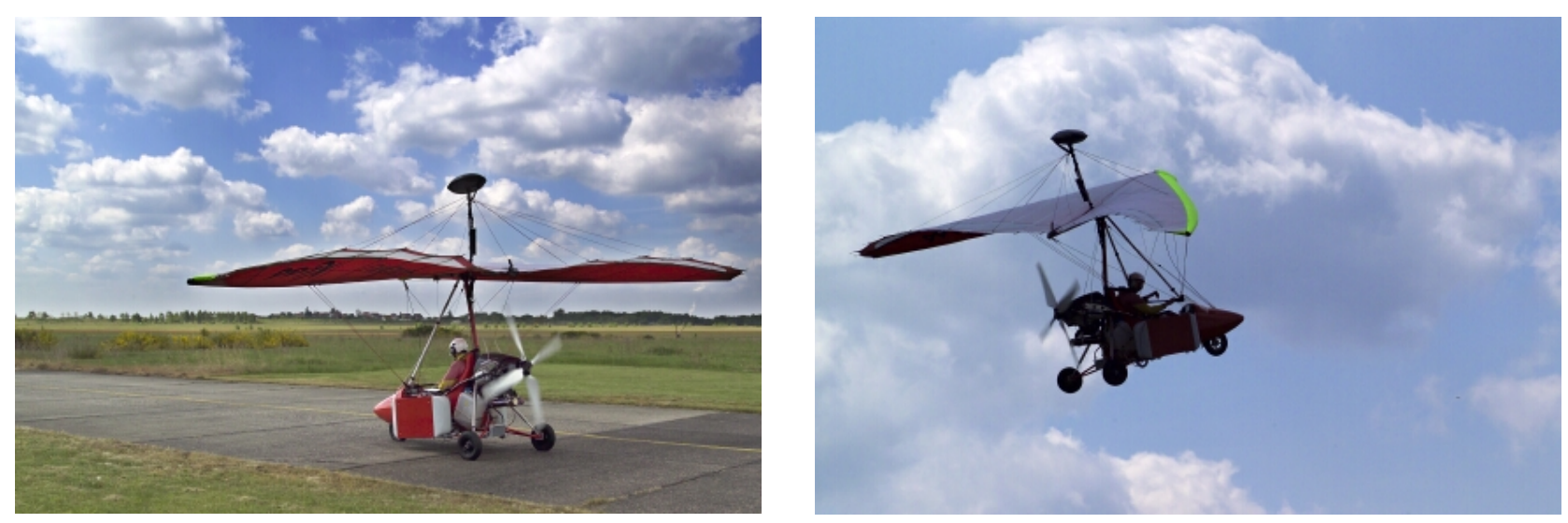

Figure 12. Operation of AVIS-2 on a microlight plane; the sensor is mounted on the both sides of the microlight (left photograph).

The data were gathered near the city of Leipzig, Germany, in 2005 within the scope of a project funded by the Centre for Environmental Research Leipzig-Halle (UFZ). The data were gathered at an altitude of $730 \mathrm{~m}$ above ground leading to a $\mathrm{GSD}_{\text {cross track }}$ of $2.4 \mathrm{~m}$ (Equation 8) and $2.2 \mathrm{~m}$ along track 
(Equation 9). The data were resampled to a spatial resolution of $2 \mathrm{~m}$. The swath width $(S W)$ can be calculated at $948 \mathrm{~m}$ using Equation 12.

$$
S W=2 h \cdot \tan \left(\frac{F O V}{2}\right)
$$

where

$$
\begin{array}{ll}
\text { FOV } & 66^{\circ}, \text { and } \\
h & \text { altitude above ground }[\mathrm{m}] .
\end{array}
$$

The area monitored in Figure 13 is located West of the city of Leipzig in a landscape dominated by opencast mining. The natural and man-made reclamation of areas affected by mine-related activities is one research theme of interest in this part of Germany. Opencast mining often gives rise to lakes. In the future these lakes will make up $25 \%$ to $30 \%$ of all standing water bodies in Germany [26]. Several mining lakes can be seen on the left part of the image surrounded by an agricultural area and small settlements which are visible on the right. The data are geometrically and radiometrically corrected. Atmospheric correction and reflectance calibration were conducted using software based on MODTRAN $4.2[6,7]$.

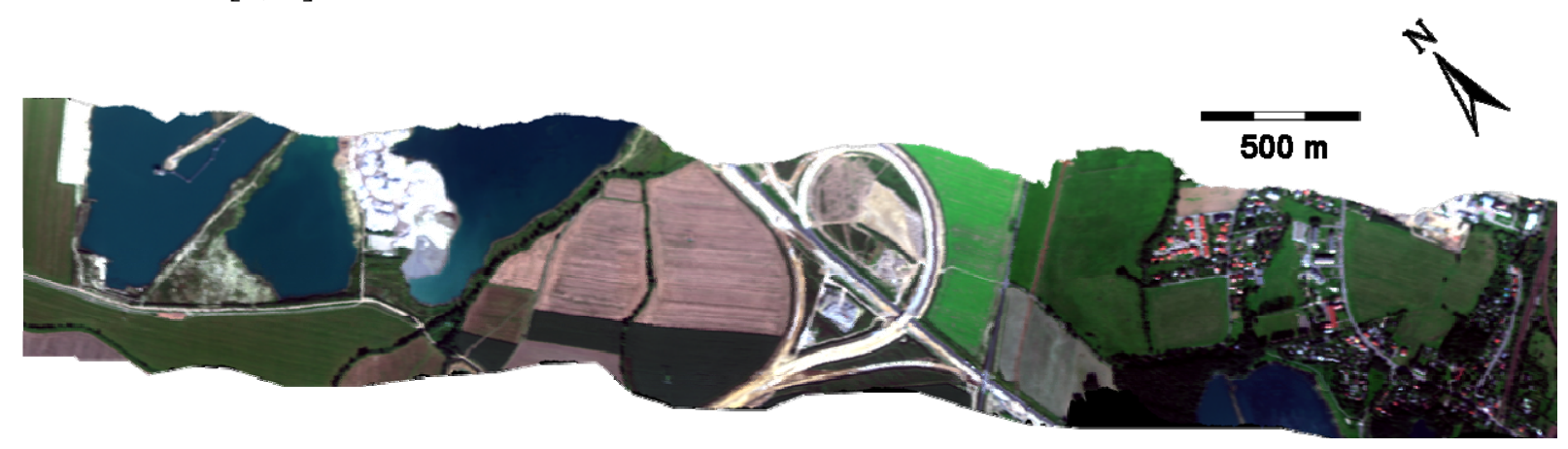

Figure 13. Geometrically and radiometrically corrected AVIS-2 image stripe acquired on August 30th 2005 [27].

Reflectance spectra of single pixels are presented in Figure 14. The selected land cover types range from surfaces such as bare soil, meadow or wooded areas to mining lakes, a soccer field or impervious areas. The spectra cover the spectral range from $411-875 \mathrm{~nm}$ and show characteristic spectral features of vegetation such as the chlorophyll absorption features in the red and the blue as well as the steep increase (red edge) towards the NIR and the NIR-plateau. Different depths of water bodies can clearly be distinguished in the VIS. The shallower the water body the more contribution of the substrate at the bottom of the lakes can be observed and the higher the reflectances are in the blue and green wavelengths [27]. 


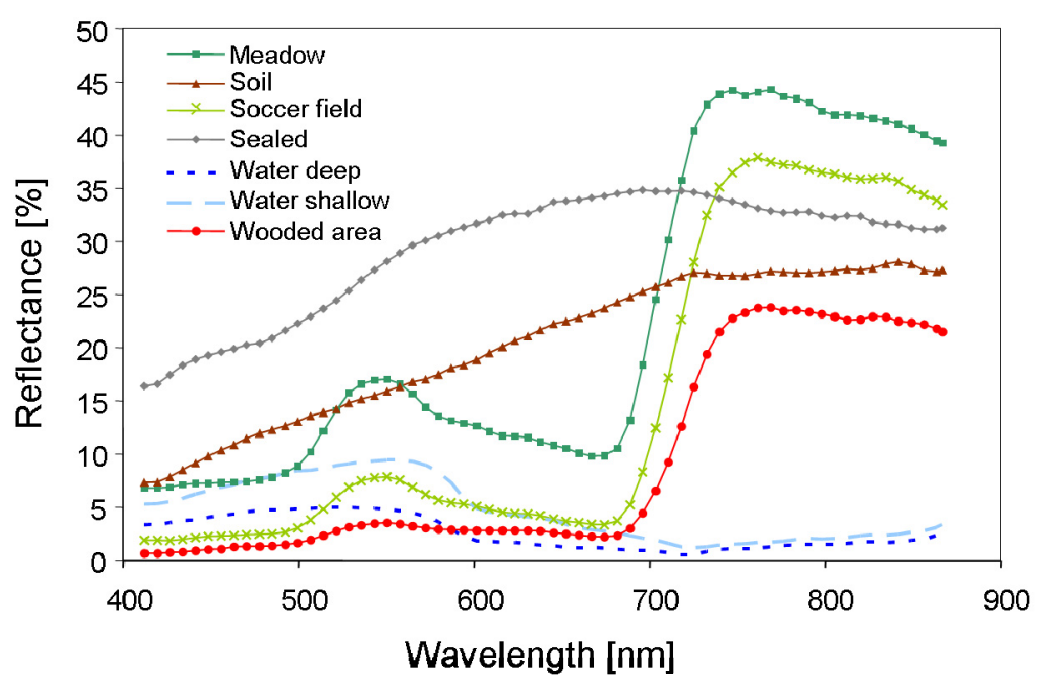

Figure 14. AVIS-2 reflectance spectra from the data shown in Figure 13 [27].

\subsection{Assessment of soil degradation in semi-arid areas}

In November 2005, AVIS-2 was operated within the scope of an airborne campaign funded by the European Space Agency (ESA), which was carried out in Southern Tunisia. The objective of the AquiferEx project was to build up a data base of multi-frequency radar (E-SAR) data as well as hyperspectral data suitable for the development and validation of science products to be developed by the project team of ESA's Aquifer [28], in particular by the companies VISTA (www.vista-geo.de) and GAF (www.gaf.de). The campaign was initiated within the scope of the TIGER (Use of Space Technology for Integrated Water Resources Management in Africa) initiative of ESA and UNESCO (United Nations Educational, Scientific and Cultural Organization) with the aim of assisting African countries to overcome problems with an integrated water resource management. Aquifer and AquiferEx had the aim in providing research and development products demonstrating the future capabilities of Earth Observation to support water management [25, 28, 29].

For the AquiferEx campaign, two test sites were selected in the Tunisian Djeffara (Figure 15), which is a sub-aquifer of the SASS (Systeme d'Aquifer du Sahara Septentrional), a transboundary ground water body extending over Algeria, Libya, and Tunisia. During the campaign, AVIS-2 was operated in parallel with the Experimental airborne Synthetic Aperture Radar system (E-SAR) by the German Aerospace Center (DLR). Both sensors were mounted on a Do-228 aircraft owned and operated by DLR. The flights were accompanied by ground measurements which were carried out contemporaneously. 


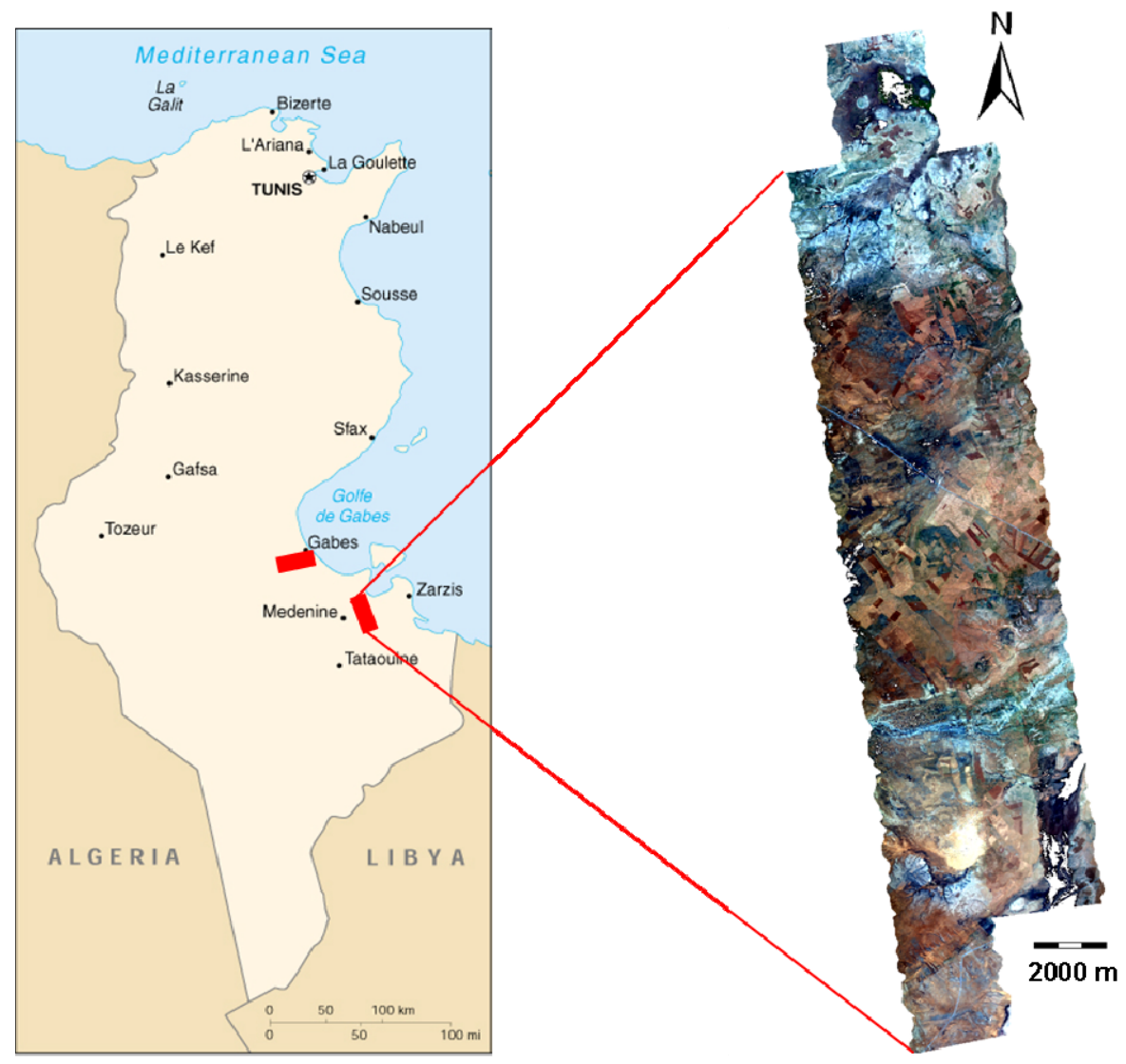

Figure 15. Location of the AquiferEx test sites in Tunisia and mosaic of three AVIS-2 flightstripes of the test site Ben Gardane (map source: http://www.lib.utexas.edu/maps/cia00/tunisia_sm00.jpg).

The test site Ben Gardane which is shown in Figure 15 is representative for the southern part of the Tunisian Djefarra and includes mainly pasture and olive plantations. About $26 \%$ of the area is grown with cereals, vegetables and forage crops, where irrigation is performed. Each year about $2.7 \mathrm{Mio} \mathrm{m}^{3}$ of water is exploited and partly used for irrigation [23].

The major problem in this region is the intrusion of sea water into the ground water body. During the last decades the pressure within the aquifer has reduced due to massive extraction of ground water for industrial and agricultural purposes leading to an invasion of salty sea water into the ground water. At the present time, the average content of salt in the ground water is $5-6 \mathrm{~g} / 1$ [23]. The salinity of the ground water and therefore of the soil, the aeolian erosion and low precipitation $(<120 \mathrm{~mm} / \mathrm{a})$ lead to land degradation [23]. Until today, the percentage affected by degradation can only be assumed. About $70 \%$ (without the Sahara desert) of Tunisia is estimated to be affected by different levels of degradation [29]. The development of a refined land use and land cover mapping using both radar and hyperspectral data were a first attempt to cope with this problem [28]. Figure 16 presents a land use classification on the basis of AVIS-2 and E-SAR data, demonstrating the high percentage of saline soil in this area. 


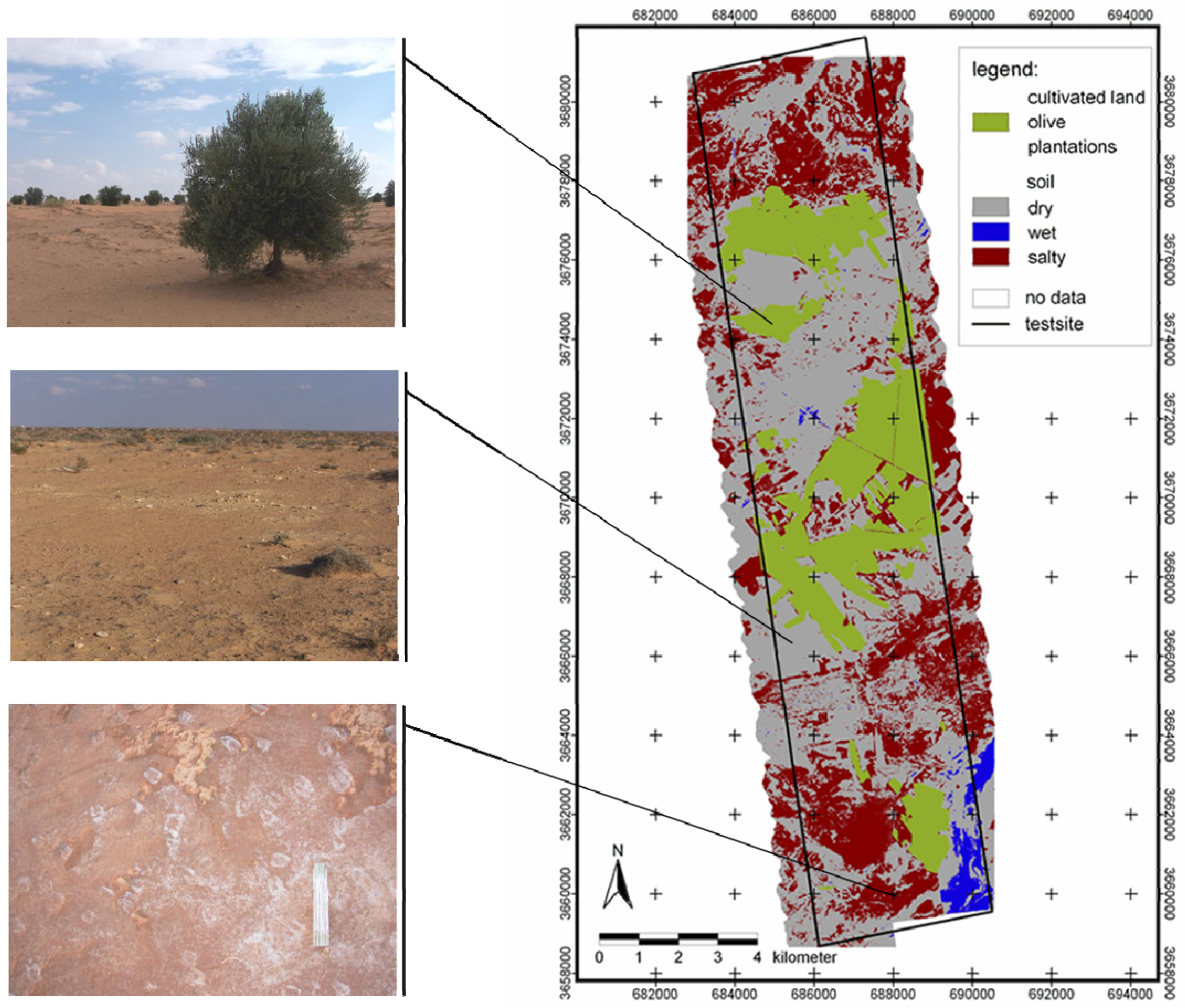

Figure 16. Land use map of Ben Gardane on the basis of AVIS-2 and E-SAR data provided by VISTA [27]. The photographs were conducted during the ground measurement campaign and show olive plantations (top), a pastoral area (center), and salt crust formation near a well (bottom) [23].

\section{Conclusions}

AVIS-2 was designed to produce high quality hyperspectral imagery for environmental monitoring purposes. The sensor was designed as an affordable tool to overcome the competing user requirements of existing airborne systems such as HYMAP, casi or AVIRIS. Therefore all components are commercially available.

The radiometric, spectral and geometric calibration and processing chains have been presented as well as exemplary AVIS-2 reflectance spectra. AVIS-2 turned out to be a valuable sensor for research and applications in a variety of fields such as agriculture, inland water studies and forestry. The specifications of AVIS- 2 are summarized in Table 1. 
Table 1. AVIS-2 specifications.

\begin{tabular}{l|l} 
Specification & AVIS-2 \\
\hline Nominal spectral range [nm] & $400-900$ \\
Analyzable spectral range [nm] & $404-875$ \\
Spectral resolution [nm] & 9 \\
Spectral sampling / resampling [nm] & $3.7 / 7.3$ \\
Radiometric sampling [bits] & 16 \\
Number of bands (oversampled / resampled) & 128 / 64 \\
Signal to Noise Ratio (SNR) [dB] & 66 at 700 nm \\
Spatial resolution [pixels per line] & 300 \\
Spatial sampling [pixels per line] & 640 \\
Field of view (FOV) [rad] & 1.16 \\
Navigation system & INS + dGPS \\
Weight [kg] & 35 \\
Period of operation [years] & since 2001
\end{tabular}

\section{Acknowledgements}

We would like to thank the Free State of Bavaria for funding the initial development of AVIS through its High Tech Initiative and the German Research Foundation (DFG) for partly funding the development of AVIS and further research. We gratefully acknowledge the support in providing AquiferEx data and results by ESA. We would like to express our gratitude to the Helmholtz Centre for Environmental Research (UFZ) Leipzig-Halle for partly funding the development of AVIS. Last but not least, many thanks are also due to Rainer Efinger for operating the system and Ingo Keding for supporting the hardware development.

\section{References}

1. Davis, C.O.; Kappus, M.; Bowles, J.; Leathers, R.A.; Korwan, D.; Downes, T.V.; Snyder, W.A.; Rhea, W.H.; Chen, W.; Fisher, J.; Bissett, W.P.; Reisse, R.A. Ocean PHILLS hyperspectral imager: design, characterization and calibration. Optics Express 2002, 10, 210-221.

2. Renecz, A.N. Remote sensing for the earth science. Wiley, New York 1999, 3, $3^{\text {rd }}$ ed.

3. Goetz, A.F.H.; Vane, G.; Solomon, J.; Rock, B.N. Imaging spectrometry for earth remote sensing. Science 1985, 228, 1147 - 1153.

4. Oppelt, N. Derivation of Chlorophyll and Nitrogen of Plants Using the Airborne Imaging Spectrometer AVIS. PhD thesis, http://edoc.ub.uni-muenchen.de/archive/00000354/ Munich 2002.

5. Spectral Imaging Ltd., ImSpector imaging spectrograph - User manual Version 2.21; 2003.

6. Berk, A.; Anderson, G.P.; Acharya, P.K; Chetwynd, J.H.; Hoke, M.L.; Bernstein, L.S.; Shettle, E.P.; Matthew, M.W.; Adler-Golden, S.M. "MODTRAN 4 Version 2 User Manual”, AIR FORCE RESEARCH LABORATORY, Space Vehicles Directorate, AIR FORCE MATERIEL COMMAND, HANSCOM AFB, 2000. 
7. Bach, H. Die Bestimmung hydrologischer und landwirtschaftlicher Oberflächenparameter aus hyperspektralen Fernerkundungsdaten (Determination of hydrological and agronomical land surface parameters using hyperspectral remote sensing data). Münchener Geographische Abhandlungen, Reihe B, Band 21, 1995.

8. Holst, G.C. CCD arrays, cameras and displays. SPIE Optical Engineering Press, Bellingham, Washington (USA), $2^{\text {nd }}$ edition, 1998.

9. Schaepman, M. Calibration of a Field Spectroradiometer. Remote Sensing Series, Vol. 31, Remote Sensing Laboratories, Dept. of Geography, University of Zurich, 1998.

10. Wolfe, W.L., Introduction to imaging spectrometers. SPIE Press, Bellingham, Washington (USA), 1997.

11. Gao, B.-C.; Montes, M.J.; Davis, C.O. Refinement of wavelength calibrations of hyperspectral imaging data using a spectrum-matching technique. Remote Sensing of Environment 2004, 90, 424433.

12. Chen, H.S. Remote sensing calibration systems. Deepak Publishing, Hampton 1997.

13. Schlaepfer, D.; Nieke, J.; Itten, K. Spatial PSF non-uniformity effects in airborne pushbroom imaging spectrometry data. IEEE Transactions on Geoscience and Remote Sensing 45 (2), 458468.

14. Nishihama, M.; Wolfe, R.; Solomon, D.; Patt, F.; Blachentte, J.; Fleig, A.; Masuoka, E. MODIS level 1A earth location algorithm theoretical basis instrument. Version 3.0, SDST-092, GSFC, 1997.

15. Oppelt, N.; Mauser, W. Hyperspectral monitoring of physiological parameters of wheat during a vegetation period using AVIS data. International Journal of Remote Sensing 2004, 25(1), 145-159.

16. Oppelt, N.; Mauser, W. Hyperspectral remote sensing - a tool for the derivation of plant nitrogen and its spatial variability within maize and wheat canopies. Stafford, J., Werner, A. (Eds.): Precision Agriculture '03. Wageningen Academic Publishers 2003, 493-498.

17. Laudien, R.; Bareth, G.; Doluschitz, G. Comparison of remote sensing based analysis of crop diseases by using high resolution multispectral and hyperspectral data - case study: Rhizoctonia solani in sugar beet. Proceedings of the $12^{\text {th }}$ International Conference on Geoinformatics Geospatial Information Research: Bridging the Pacific and Atlantic 7-9 June 2004, Gävle (Sweden), 670-676.

18. Schmidtlein, S.; Sassin, J. Mapping of continuous floristic gradients in grasslands using hyperspectral imagery. Remote Sensing of Environment 2004, 92, 126-138.

19. Begiebing, S.; Schneider, M.; Bach, H.; Wagner, P. Assessment of in-field heterogeneity for determination of the economic potential of precision farming. Stafford, J., Werner, A. (Eds.): Precision Agriculture '07. Wageningen Academic Publishers 2007, 811-818.

20. Schmidtlein, S. Imaging spectroscopy as a tool for mapping Ellenberg indicator values. Journal of Applied Ecology 2005, 42, 966-974.

21. Schmidtlein, S.; Zimmermann, P.; Schüpferling, R.; Weiss, C. Mapping the floristic continuum: Ordination space position estimated from imaging spectroscopy. Journal of Vegetation Sciences 2007, 18, 131-140.

22. Bach, H. Makrophytenklassifikation auf Grundlage von Satellitenbildern am Beispiel des Steinhuder Meeres. URL: http://vista-geo.de/pdf/gewaessermonitoring.pdf (28 August 2007). 
23. Scheiber, R.; Oppelt, N.; Mauser, W. AquiferEx Optical and Radar Campaign - Experiment Plan. European Space Agency ESRIN. 2005.

24. Scheiber, R.; Oppelt, N.; Horn, R.; Hajnsek, I.; Ben Khadhra, K.; Keller, M.; Wegscheider, S.; Mauser, W.; Bianchi, R. AquiferEx Optical and Radar Campaign: Objectives and First Results. Proc. of 6th European Conference on Synthetic Aperture Radar, May 16-18, 2006, Dresden (Germany), published on CD-Rom ISBN 3-8007-2960-1.

25. Scheiber, R.; Hajnsek, I.; Horn, R.; Oppelt, N.; Mauser, W.; Ben Baccar, B.; Bianchi, R. AquiferEx: Results of the optical and radar airborne campaign in Tunisia. Proceedings of the POLinSar Workshop, 22-24 January, 2007, ESA/ESRIN Frascati (Italy), forthcoming.

26. Boehrer, B.; Matzinger, A.; Schimmele, M. Similarities and Differences in the Annual Temperature Cycles of East German Mining Lakes. Limnologica, 30, 2000, 271-279.

27. Oppelt, N., Mauser, W., Three generations of the airborne imaging Spectrometer AVIS expectations, applications, results. Proceedings of SPIE conference "Europhotonics Europe", Strasbourg (France), 02.04.-07.04.2006, Vol. 6189, CD-Rom publication ISBN 97-80819462459.

28. Gesellschaft für Angewandte Fernerkundung (GAF), Aquifer - Earth observation in support for transboundary aquifer management. URL: http://www2.gaf.de/aquifer/ (10 September 2007).

29. European Space Agency (ESA), TIGER Initiative - Looking after water in Africa. URL:http://www.tiger.esa.int/ (10 September 2007).

30. Mtimet, A., Soils of Tunisia. Options de Mèditerranéennes, Séries B, 34, 2001, 243-268.

(C) 2007 by MDPI (http://www.mdpi.org). Reproduction is permitted for noncommercial purposes. 